\title{
Author Correction: Directed species loss reduces community productivity in a subtropical forest biodiversity experiment
}

Yuxin Chen, Yuanyuan Huang (D), Pascal A. Niklaus (D), Nadia Castro-Izaguirre, Adam Thomas Clark, Helge Bruelheide (D), Keping Ma and Bernhard Schmid (D)

Correction to: Nature Ecology \& Evolution https://doi.org/10.1038/s41559-020-1127-4, published online 2 March 2020.

In the version of this Article originally published, two instances of the word 'decreased' should have been 'increased'.

In the fourth paragraph of the Results subsection 'Effects of species loss on productivity', the sentence "Mean pairwise similarities between species in postextinction communities for SLA and ED decreased faster..." should have read "Mean pairwise similarities between species in postextinction communities for SLA and ED increased faster...”

In the fifth paragraph of the Discussion section, the sentence "We found that species similarities in SLA and ED decreased faster..." should have read "We found that species similarities in SLA and ED increased faster...".

These errors have now been corrected.

Published online: 13 March 2020

https://doi.org/10.1038/s41559-020-1174-X

(c) The Author(s), under exclusive licence to Springer Nature Limited 2020 